\title{
A Method for Converting Colors for Color-impaired People, Considering Saturation and Contrast Ratio
}

\author{
Junko Shirogane \\ Miho Saito
Waseda University
}

Tokyo Woman's Christian University

\author{
Yuko Iwase \\ Waseda University \\ Yoshiaki Fukazawa \\ Waseda University
}

\author{
Hajime Iwata \\ Kanagawa Institute of Technology
}

\begin{abstract}
Because color-impaired people have difficulty in perceiving specific colors, colors used in documents must be converted for them. The colors of documents include the intentions of their designers. That is, designers use different colors for parts of the document that they want to be attractive or distinguishable in some way. In existing conversion methods, however, colors are not converted based on a consideration of the designers' intentions. Hence, we propose a method for converting document colors for color-impaired people in a manner that takes the designers' intentions into account. Here, we define the designers' intentions as "attractiveness" and "distinguishability." In our method, the original colors are classified into three levels, low, middle, and high, based on their saturation. In each saturation level, some colors are defined as available for conversion. Next, the original colors are converted to colors in the corresponding saturation levels. According to the RGB (red-green-blue) values of the original colors, the target colors for conversion can be specified. Then, the contrast ratios of the pair of foreground and background colors are calculated. If the contrast ratios do not satisfy the specific thresholds, the colors are additionally converted to other colors in the corresponding saturation levels. Finally, when the contrast ratios satisfy the specific thresholds, the conversion is complete.
\end{abstract}

\section{INTRODUCTION}

$\mathbf{V}$ ARIOUS colors are used in artifacts such as computer displays, prints, and devices. The effectiveness of color in user interface aesthetics, attractiveness, and distinguishability is well known. Therefore, the usage of color is quite important in the design of artifacts. However, color-impaired people perceive color differently from people with full color vision. Specifically, they have difficultly distinguishing between red and green or blue and yellow. Additionally, some people have difficulty perceiving colors at all. Color impairments include both congenital cases and acquired cases due to advanced age and illnesses. Men have color impairments more frequently than women. Although the rates of color impairment differ among ethnicities, the frequencies of color impairment are about $2-10 \%$ for men and $0.2-0.5 \%$ for women [1]. Accordingly, approximately 200 million people worldwide have color impairments, nearly equal to the number of men with blood type AB. To make artifacts perceivable for color-impaired people, the colors of artifacts are often converted.

To convey information, attractiveness and distinguishability are important. Generally, colors with high saturation and luminance have high attractiveness. Designers often use colors with high attractiveness for artifact contents that they wish to emphasize. Meanwhile, background and foreground colors affect distinguishability. If the contrast ratio of relative luminance between the background and foreground colors of the contents of an artifact is low, the distinguishability of the contents will also be low. Regardless of color vision, distinguishability can be provided by ensuring that the contrast ratios between background and foreground colors are sufficiently high. Although many methods have been proposed for converting the original colors of artifacts to those that color-impaired people can easily perceive, in many cases the intentions of the artifact designers are not considered.

The purpose of our method is to convert original colors for color-impaired people in such a way that they can perceive the designers' intentions. Here, the designers' intentions are defined as "attractiveness" and "distinguishability." To convert colors, we focus on saturation and contrast ratios. In advance, we create a basic conversion table. For this purpose, colors that color-impaired people can easily perceive, selected by the Color Universal Design Organization (CUDO) of Japan [2], are classified into three saturation levels. For conversions of original colors, first, the saturations of the colors are calculated, and the saturation levels are identified. Second, the original colors are converted to the closest colors in the same saturation levels of the basic conversion table. Third, the contrast ratios of pairs of background and foreground colors are calculated. Finally, if the calculated values do not satisfy specific thresholds, the converted colors are additionally converted to other colors in the same saturation levels. Hereafter, the original colors that designers use are called "original colors," the converted colors based on the basic conversion table in the second step are called "provisional colors," and the ultimate converted colors based on the contrast ratios are called "final colors." In addition, the current targets of our method are limited to text, rather than the images in documents.

The rest of this paper is organized as follows. Section II describes related works. Section III summarizes the background of this research, while Section IV shows the features. Section $\mathrm{V}$ describes the details of strategies for converting colors. Section VI describes how to convert colors in documents. Section VII provides an evaluation of our method. Section VIII concludes this paper. 
TABLE I

TYPES OF COLOR VISION

\begin{tabular}{|l|l|l|l|}
\hline Types of color visions & Cone conditions & Descriptions \\
\hline Common & C-type & All three cones are normal & All colors can be perceived \\
\hline $\begin{array}{l}\text { Color } \\
\text { Impairments }\end{array}$ & P-type (Strong) & Lack of L-cones & \multirow{3}{*}{ Red and green colors are difficultly perceived } \\
\cline { 2 - 3 } & P-type (Weak) & Shift of spectral sensitivities of L-cones & \\
\cline { 2 - 3 } & D-type (Strong) & Lack of M-cones & \\
\cline { 2 - 3 } & D-type (Weak) & Shift of spectral sensitivities of M-cones & \\
\cline { 2 - 4 } & T-type & Lack of S-cones & Blue and yellow colors are difficultly perceived \\
\cline { 2 - 3 } & A-type & Lack of two cone types or all cones & Colors can not be perceived \\
\hline
\end{tabular}

\section{RELATED WORKS}

To make artifact colors perceivable to color-impaired people, the original colors are often converted for them, and many studies related to color conversion have been performed.

For converting document colors, Zhau et al. proposed a method for converting colors of web pages in a mobile environment [3]. Their purpose was to reduce the costs of selecting colors for conversion. To this end, a conversion method [4] was extended, pairs of background and foreground colors were extracted, and colors to be converted were limited by color pairs. In this manner, the costs of computational assessment of color conversion could be reduced. Then, colors were converted based on a model of users' subjective judgment of colors [5]. Although this method was targeted to text in documents, document structures were not considered.

To convert targets other than documents, Takimoto et al. proposed a method for converting image colors so that even people with full color vision would feel that the converted images were natural [6]. First, the number of colors was reduced using a median cut algorithm [7]. Next, saturation and lightness were modified using a modified Color2Gray algorithm [8]. In this case, because the converted colors sometimes became out of ranges of color gamut, color correlation was performed. A study by Wahab et al. aimed to convert image colors for people with protanope impairments [9]. First, colors in RGB (red-green-blue) color space were converted to LMS (long-medium-short) color space. Second, the LMS information that people with protanope impairments could not perceive were deleted from the LMS values. Third, the contrast between red and blue was increased. Meanwhile route maps and road signs were targeted using a multilayer neural network [10]. This network consisted of (1) layers of color conversion of images, (2) layers of perceptual models for color impairments, and (3) layers of color discrimination. Strategies of color conversion were determined by (1), perceptual models of color-impaired people were simulated by (2), and color edges of images for color-impaired people were simulated by (3). Color vision of color-impaired people was simulated by converting RGB color spaces to CIE XYZ [11][12] color spaces. $\mathrm{X}, \mathrm{Y}$, and $\mathrm{Z}$ values were calculated from red, green, and blue values [13], and reaction values of LMS were calculated from the $\mathrm{X}, \mathrm{Y}$, and $\mathrm{Z}$ values. Then, the LMS values of simulations for people with protanope and deuteranope impairments were calculated [14][15]. Layers (2) and (3) were fixed, and layers (1) were trained to determine color- conversion rules. In addition, Harwahyu et al. proposed a method for converting colors in embedded systems and smart phones so that color-impaired people can understand display colors [16]. First, colors on the display were represented by 16 color names. Users selected colors on the display, and the HSL (hue-saturation-lightness) values were calculated. Then, by changing hue and lightness values, colors were adjusted for the color-impaired users. In this manner, many color conversion methods for images were provided, but the designers' intentions were not focused on.

In study of color perception by color-impaired people, Tajima et al. quantified and visualized information that could be obtained by differences in color vision [17]. They then defined "visual salience" as "visual conspicuity predicted from image features." First, visual salience was modeled using images. Image colors were converted step by step, and loss/gain information was predicted by comparing models. Then, psychophysical experiments were performed, and subjective differences between the predictions and results of experiments were investigated. Correlations were observed. Meanwhile, color use guidelines for people with protanope and deuteranope impairments were provided based on scientific data [18]. Strategies for representing data were classified into five types, and guidelines were developed for individual classifications. These approaches can support determination of colors for color-impaired people; however, concrete strategies for converting colors of existing documents were not provided.

\section{PRELIMINARY}

\section{A. Types of color vision}

Due to differences in retinas among individual people, the colors that people see are different. Consequently, various types of color visions exist. There are two types of visual cells in human retinas, rod cells and cone cells. Rod cells work in the dark, whereas cone cells work in the light. Cone cells consist of L-cones, M-cones, and S-cones, which perceive red, green, and blue light, respectively. Color vision differs among individuals based on the presence or absence of these three types of cones, as well as whether their spectral sensitivities work normally. Accordingly, color vision can be classified into five types, as shown in Table I.

People that have all three types of cones are common type (C-type), whereas others with a subset of the three types of cones, or in whom the spectral sensitivities of cones are shifted, have color impairments. People who do not have 
L-cones or in whom the spectral sensitivities of L-cones are shifted toward those of $\mathrm{M}$-cones are protanope (P-type), whereas people who do not have M-cones or in whom the spectral sensitivities of M-cones are shifted toward those of Lcones are deuteranope (D-type). People with P-type or D-type impairments have difficulty perceiving differences between red and green colors. Meanwhile, people who do not have Scones are tritanope (T-type), and have difficultly perceiving differences between blue and yellow colors. People who have only one or no cones are achromat (A-type), and perceive only light and darkness. The most common types are P-type and D-types, whereas T-type is rare [1].

\section{B. Color universal design}

Color-universal design refers to designs in which colors of artifacts are adjusted so that people can perceive colors regardless of their color vision status. Such designs are not only for color-impaired people, but also for people with normal color vision. Many studies have been conducted, such as [19][20][21], and many governments and companies have developed guidelines for color-universal design.

\section{FEATURES OF OUR METHOD}

\section{Representation of designers' intentions}

Generally, colors with high saturation have high attractiveness. Designers often use colors with high saturation for content that they want to emphasize. Meanwhile, when contrast ratios of relative luminance between background and foreground colors are high or low, the readability and distinguishability will be correspondingly high or low. When designers want to make contents readable or distinguishable, they use a pair of colors for which the contrast ratio is high. That is, designers represent intentions regarding the contents that they do or do not want to emphasize or make readable/distinguishable by the colors. In our method, colors can converted in a manner that reflects the designers' intentions.

\section{Conversion strategy for documents}

As mentioned in Section I, our method is targeted at text in documents. For text, background and foreground colors in documents do not always correspond in a one-to-one manner. In some cases, multiple foreground colors are used for one background color. If background and foreground colors are converted as pairs in a certain part of the document, the contrast ratios of relative luminance between background and foreground colors in other parts of the document may not satisfy the thresholds. Thus, in our method, the combinations of background and foreground colors are analyzed. Background and foreground colors are not converted as pairs. Instead, as necessary, background (foreground) colors are fixed, and only the foreground (background) colors are converted.

\section{STRATEgIES FOR COLOR CONVERSION}

Color conversions are performed in two steps. First, colors are converted based on a basic conversion table. After that, the contrast ratios of relative luminance between background and foreground colors are calculated. If the contrast ratios do not satisfy specific thresholds, additional conversions are performed. In this section, we describe the details of the basic conversion table and two conversion steps.

\section{A. Basic conversion table}

The basic conversion table is given by our method in advance. CUDO in Japan selects 20 colors that color-impaired people can easily perceive [2]. In this paper, these colors are called "recommended colors." Using the recommended colors, the basic conversion table is created. The purpose of creating the basic conversion table is to ensure that the color saturations are not significantly altered before and after conversion. Using this table, colors can be converted to other colors with almost the same saturation levels.

To create the basic conversion table, first, the HSV (huesaturation-value) values of the recommended colors are calculated using their RGB values by formulas (1)-(3) [22]. According to these formulas, because colors are represented by values of hue $(\mathrm{H})$, saturation $(\mathrm{S})$, and brightness $(\mathrm{V})$, the saturation values of the colors can be obtained. In formulas (1)-(3), R, G, and B indicate the red, green, and blue values of the RGB value for a color, whereas "Max" and "Min" indicate the maximum and minimum values among the $\mathrm{R}, \mathrm{G}$, and $\mathrm{B}$ values. These formulas operate on values as decimal numbers.

$$
\begin{aligned}
& H= \begin{cases}\text { Notdefined } & , \text { Max }=\text { Min } \\
60 \times \frac{G-B}{\text { Max }- \text { Min }} & , \text { Max }=R \\
60 \times \frac{B-R}{\text { Max-Min }}+120 & , \text { Max }=G \\
60 \times \frac{R-G}{\text { Max-Min }}+240 & , \text { Max }=B\end{cases} \\
& S= \begin{cases}\text { Notdefined , Max }=0 \\
\frac{\text { Max }- \text { Min }}{\text { Max }, \text { Max } \neq 0}\end{cases} \\
& V=\text { Max }
\end{aligned}
$$

Next, the recommended colors are classified into three saturation levels, low, middle, and high, according to their saturation values based on the Practical Color Coordinate System (PCCS) [23][24]. According to the PCCS, the calculated saturation values from 0 to 1 based on formula (2) are divided into 10 levels (0s-9s) at regular intervals. 0s indicates achromatic color, $1 \mathrm{~s}-3 \mathrm{~s}$ indicate low saturation level, $4 \mathrm{~s}-6 \mathrm{~s}$ indicate middle saturation level, and 7s-9s indicate high saturation level. As specified by the PCCS, 0s is added to the low saturation level; thus, three saturation levels are defined in our method. That is, the saturation values of colors from 0 to 0.33 are classified into the low saturation level, colors from 0.34 to 0.67 into the middle saturation level, and colors from 0.68 to 1 into the high saturation level.

Next, recommended colors in each saturation level are sorted according to their color codes. Finally, all colors 
TABLE II

BASIC CONVERSION TABLE

\begin{tabular}{|c|c|c|c|}
\hline Saturation before conversion & Color codes before conversion & Conversion candidate 1 & Conversion candidate 2 \\
\hline \multirow{6}{*}{$\begin{array}{l}\text { Low } \\
\text { (Saturation value from } 0 \text { to } 0.33 \text { ) }\end{array}$} & (1) \#000000 - \#7F878F & $\# 000000$ & \#7F878F \\
\hline & (2) \#7F878F - \#B4EBFA & \#7F878F & \#B4EBFA \\
\hline & (3) \#B4EBFA - \#C7B2DE & \#B4EBFA & \#C7B2DE \\
\hline & (4) \#C7B2DE - \#C8C8CB & \#C7B2DE & \#C7B2DE \\
\hline & (5) \#C8C8CB - \#FFD1D1 & \#C8C8CB & \#FFD1D1 \\
\hline & (6) \#FFD1D1 - \#FFFFFF & \#FFD1D1 & \#FFFFFF \\
\hline \multirow{8}{*}{$\begin{array}{l}\text { Middle } \\
\text { (Saturation value from } 0.34 \text { to } 0.67 \text { ) }\end{array}$} & (7) $\# 000000$ - \#35A16B & \#35A16B & $\mathrm{n} / \mathrm{a}$ \\
\hline & (8) \#35A16B - \#66CCFF & \#35A16B & \#66CCFF \\
\hline & (9) \#66CCFF - \#99E7B0 & \#66CCFF & \#99E7B0 \\
\hline & (10) \#99E7B0 - \#CBF266 & \#99E7B0 & \#CBF266 \\
\hline & (11) \#CBF266 - \#EDC58F & \#CBF266 & \#EDC58F \\
\hline & (12) \#EDC58F - \#FF99A0 & \#EDC58F & \#FF99A0 \\
\hline & (13) \#FF99A0 - \#FFFF99 & \#FF99A0 & \#FFFF99 \\
\hline & (14) \#FFFF99 - \#FFFFFF & \#FFFF99 & $\mathrm{n} / \mathrm{a}$ \\
\hline \multirow{7}{*}{$\begin{array}{l}\text { High } \\
\text { (Saturation value from } 0.68 \text { to } 1 \text { ) }\end{array}$} & (15) \#000000 - \#0041FF & $\# 0041 \mathrm{FF}$ & $\mathrm{n} / \mathrm{a}$ \\
\hline & (16) \#0041FF - \#663300 & $\# 0041 \mathrm{FF}$ & $\# 663300$ \\
\hline & (17) \#663300 - \#9A0079 & $\# 663300$ & \#9A0079 \\
\hline & (18) \#9A0079 - \#FAF500 & \#9A0079 & \#FAF500 \\
\hline & (19) \#FAF500 - \#FF2800 & \#FAF500 & \#FF2800 \\
\hline & (20) \#FF2800 - \#FF9900 & \#FF2800 & \#FF9900 \\
\hline & (21) \#FF9900 - \#FFFFFF & \#FF9900 & $\mathrm{n} / \mathrm{a}$ \\
\hline
\end{tabular}

from color code \#000000 to \#FFFFFF (hexadecimal notation) are divided by the color codes of the sorted recommended colors. That is, the color codes of the sorted recommended colors (\#7F878F, \#B4EBFA, etc.) are used as delimiters, and several divisions are created (divisions of \#000000 \#7F878F, \#7F878F - \#B4EBFA, etc.). This manipulation is performed in all three saturation levels. The first and last colors in each division are defined as conversion candidates 1 and 2, respectively. The reason for defining two conversion candidates is that the original colors of the document should be converted to the closest possible colors. However, for the first and last division of colors in the middle and high saturation levels, one of conversion candidates becomes either $\# 000000$ or \#FFFFFF. Because these color codes are achromatic, they are not appropriate conversion candidates for original colors in the middle and high saturation levels. Therefore, colors of \#000000 and \#FFFFFF are removed from the conversion candidates in the middle and high saturation levels. The resultant basic conversion table is shown in Table II.

\section{B. Conversion strategy}

First, original colors are converted using the basic conversion table to provisional colors. Next, the contrast ratios between background and foreground colors are calculated. Then, if the calculated contrast ratios do not satisfy specific thresholds, the provisional colors are additionally converted, and ultimately the final colors are determined.

1) Conversion by basic conversion table: First, the saturation value of an original color is calculated using formula (2). Next, the saturation level of the original color is determined using the calculated saturation value. Then, the corresponding divisions in the saturation level to the original color is determined. Finally, differences in R, G, and B values between the original color and two conversion candidates in the division are calculated using formula (4). In this formula, "Diff" indicates the difference. $R_{o}, G_{o}$, and $B_{o}$ indicate the $\mathrm{R}, \mathrm{G}$, and B values of the original color, whereas $R_{c}, G_{c}$, and $B_{c}$ indicate the $\mathrm{R}, \mathrm{G}$, and $\mathrm{B}$ values of the one of the candidate colors. After calculating the differences, the conversion candidate for which the difference is smaller is determined as the provisional color. Figure 1 shows the workflow for this determination.

$$
\operatorname{Diff}=\left|R_{o}-R_{c}\right|+\left|G_{o}-G_{c}\right|+\left|B_{o}-B_{c}\right|
$$

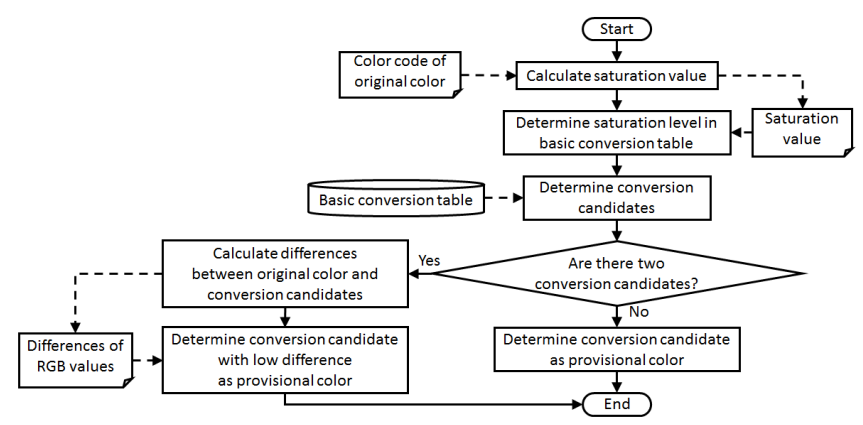

Fig. 1. Workflow of conversion of original color

As examples, original colors \#B6B7C6 (example 1-1), \#E8F2B0 (example 1-2), and \#004082 (example 1-3) are converted. For example 1-1, the calculated saturation value according to formula (2) is 0.08 . This saturation value is in the low range of saturation level in the basic conversion table (Table II). Accordingly, conversion candidates range from division (1) to division (6). According to the original color code, the original color is in the range of division (3). Thus, the conversion candidates are limited to \#B4EBFA and \#C7B2DE. Similarly, the saturation values of colors \#E8F2B0 
TABLE III

EXAMPLES OF DIFFERENCE CALCUATIONS

\begin{tabular}{|c|c|c|}
\hline & Example 1-1 & Example 1-2 \\
\hline \multirow[t]{3}{*}{ RGB value } & Original (\#B6B7C6) $\quad\left(R_{o}: 182, G_{o}: 183, B_{o}: 198\right)$ & $\left(R_{o}: 232, G_{o}: 242, B_{o}: 176\right)$ \\
\hline & $\left(R_{c}: 180, G_{c}: 235, B_{c}: 250\right)$ & $\left(R_{c}: 200, G_{c}: 200, B_{c}: 203\right)$ \\
\hline & \#C7B2DE $\quad\left(R_{c}: 199, G_{c}: 178, B_{c}: 222\right)$ & $\left(R_{c}: 255, G_{c}: 209, B_{c}: 209\right)$ \\
\hline \multirow[t]{2}{*}{ Diff } & \#B4EBFA $\quad:|182-180|+|183-235|+|198-250|=106$ & $:|232-200|+|242-200|+|176-203|=101$ \\
\hline & \#C7B2DE $\quad:|182-199|+|183-178|+|198-222|=46$ & $:|232-255|+|242-209|+|176-209|=89$ \\
\hline
\end{tabular}

(example 1-2) and \#004082 (example 1-3) are 0.27 and 1.00, respectively. According to their saturation values, \#E8F2B0 (example 1-2) and \#004082 (example 1-3) are in the low and high saturation levels, respectively. Next, the division of color \#E8F2B0 (example 1-2) is determined as (5) in Table II, yielding conversion candidates \#C8C8CB and \#FFD1D1. The division of the color \#004082 (example 1-3) is determined as (15); thus, the only conversion candidate is \#0041FF. The colors in examples 1-1 and 1-2 have two conversion candidates each, whereas the color in example 1-3 has only one. Thus, one of the candidates from each of examples 1-1 and 1-2 must be selected, whereas the conversion candidate of example 1-3 is determined as the provisional color.

TABLE IV

EXAMPLES OF COLOR CONVERSION TO PROVISIONAL COLOR

\begin{tabular}{|l|c|c|}
\hline & Original color & Provisional color \\
\hline Example & Color code: \#B6B7C6 & Color code: \#C7B2DE \\
$1-1$ & Saturation value: 0.08 & Saturation value: 0.20 \\
\hline Example & Color code: \#E8F2B0 & Color code: \#FFD1D1 \\
$1-2$ & Saturation value: 0.27 & Saturation value: 0.18 \\
\hline Example & Color code: \#004082 & Color code: \#0041FF \\
$1-3$ & Saturation value: 1.00 & Saturation value: 1.00 \\
\hline
\end{tabular}

For examples 1-1 and 1-2, the differences in R, G, and $\mathrm{B}$ values between the original and conversion colors are calculated using formula (4). The R, G, B values of examples 1-1 and 1-2 and the difference calculations are shown in Table III. For simplicity, the values are shown as decimal numbers. As a result, for example 1-1, the difference for color \#C7B2DE is smaller than that for \#B4EBFA; consequently, the color \#C7B2DE is determined as the provisional color for \#B6B7C6. For example 1-2, the difference for color \#FFD1D1 is smaller than that for \#C8C8CB; consequently color \#FFD1D1 is determined as the provisional color of \#E8F2B0. Table IV shows the conversion results for these three examples.

2) Calculation of contrast ratio: After determination of provisional colors, the contrast ratios of relative luminance between background and foreground colors are calculated. If the contrast ratios do not satisfy specific thresholds, additional conversions are performed.

To calculate the contrast ratios, relative luminance values are calculated using formulas (5)-(7) [25]. The relative luminance values range from 0 to 1 . The colors \#000000 and \#FFFFFF are the darkest and the lightest, respectively, with relative luminance values are 0 and 1 , respectively. In this formula,
" $L_{R}$ ", " $L_{G}$ ", " $L_{B}$ " indicate the relative luminance for the red, green, and blue elements of a color, respectively. " $R$ " indicates the red value of the color, and " $L$ " indicates the calculated relative luminance. Although formulas (5) and (6) show the calculations for obtaining $L_{R}$ for the red element of the color, $L_{G}$ and $L_{B}$ for green and blue elements can be calculated similarly. These formulas calculate values as decimal numbers.

$$
\begin{gathered}
R s R G B=\frac{R}{255} \\
L_{R}= \begin{cases}\frac{R}{12.92}, & R s R G B \leq 0.03928 \\
\left(\frac{R s R G B+0.055}{1.055}\right)^{2.4}, & R s R G B>0.03928\end{cases} \\
L=0.2126 \times L_{R}+0.7152 \times L_{G}+0.0722 \times L_{B}
\end{gathered}
$$

After calculating the relative luminance of original colors, the contrast ratios between background and foreground colors are calculated using formula (8) [25]. For a pair of background and foreground colors, $L_{1}$ indicates the relative luminance value of the lighter color (the greater value), whereas $L_{2}$ indicates the relative luminance value of the darker color (the smaller value). $C R$ indicates the calculated contrast ratio.

$$
C R=\frac{L_{1}+0.05}{L_{2}+0.05}
$$

3) Additional conversion: When the contrast ratios of relative luminance between background and foreground colors are high, readability and distinguishability are also high. Thus, designers use a pair of background and foreground colors with a high contrast ratio to make contents readable and distinguishable, whereas they use a pair of background and foreground colors of contents with low contrast ratios when they do not want to make contents distinguishable. In addition, according to accessibility guidelines issued by the World Wide Web Consortium (W3C) [25], to ensure readability of documents, the contrast ratios between background and foreground colors should be greater than 4.5. Considering these factors, in our method, when the calculated contrast ratios in Section V-B2 are smaller than 4.5 and smaller than the contrast ratios of the pair of original background and foreground colors, the provisional colors must be additionally converted.

In the additional conversion, the colors in the saturation levels (Table II) of the background and foreground colors 
TABLE V

EXAMPLES OF DETERMINING FINAL COLORS

\begin{tabular}{|c|c|c|c|c|c|c|}
\hline & \multicolumn{2}{|c|}{ Original color } & \multicolumn{2}{|c|}{ Provisional color } & \multicolumn{2}{|c|}{ Final color } \\
\hline $\begin{array}{l}\text { Example } \\
2-1\end{array}$ & Sample & $\begin{array}{l}\text { Background color: \#FFE6E7 } \\
\text { Foreground color: \#CC0000 } \\
\text { Contrast ratio: } 4.97\end{array}$ & Sample & $\begin{array}{l}\text { Background color: \#FFD1D1 } \\
\text { Foreground color: \#9A0079 } \\
\text { Contrast ratio: } 5.77\end{array}$ & Sample & $\begin{array}{l}\text { Background color: \#FFD1D1 } \\
\text { Foreground color: \#9A0079 } \\
\text { Contrast ratio: } 5.77\end{array}$ \\
\hline $\begin{array}{l}\text { Example } \\
2-2\end{array}$ & Sample & $\begin{array}{l}\text { Background color: \#EAE6E7 } \\
\text { Foreground color: \#6E6C6C } \\
\text { Contrast ratio: } 4.22\end{array}$ & Sample & $\begin{array}{l}\text { Background color: \#FFD1D1 } \\
\text { Foreground color: \#7F878F } \\
\text { Contrast ratio: } 2.65\end{array}$ & Sample & $\begin{array}{l}\text { Background color: \#B4EBFA } \\
\text { Foreground color: \#000000 } \\
\text { Contrast ratio: } 16.2\end{array}$ \\
\hline $\begin{array}{l}\text { Example } \\
2-3\end{array}$ & Sample & $\begin{array}{l}\text { Background color: \#1E4611 } \\
\text { Foreground color: \#66FF33 } \\
\text { Contrast ratio: } 8.20\end{array}$ & & $\begin{array}{l}\text { Background color: \#663300 } \\
\text { Foreground color: \#663300 } \\
\text { Contrast ratio: } 1\end{array}$ & Sample & $\begin{array}{l}\text { Background color: \#663300 } \\
\text { Foreground color: \#FAF500 } \\
\text { Contrast ratio: } 8.89\end{array}$ \\
\hline
\end{tabular}

are sorted again according to relative luminance. Then, for a pair of background and foreground colors, the dark color (the color with the smaller value of relative luminance) and the light color (the color with the greater value of relative luminance) are shifted to the darker color and the lighter color in the corresponding saturation levels, respectively, one by one, and then the contrast ratio is calculated again. This process is repeated until the contrast ratio becomes greater than the threshold. The threshold is the contrast ratio of the pair of original background and foreground colors. However, if the shifted colors fall out of the ranges of the saturation levels in Table II determined in Section V-B1, the attractiveness of the original colors cannot be maintained, and the designers' intentions cannot be perceived by color-impaired people. Thus, even though the contrast ratio of a pair of background and foreground colors does not become greater than the threshold, the shifting of colors is stopped when both background and foreground colors become the first and the last colors in the corresponding saturation levels. When the shifting of colors is stopped, the shifted colors are considered to be the final colors.

Table V shows examples of conversion of original colors to final colors. In this table, the original colors are converted to provisional colors by the process of Section V-B1.

For example 2-1, the provisional colors of background and foreground are \#FFD1D1 and \#9A0079, respectively, and the contrast ratio of the colors are 5.77 and $>4.97$ (threshold for the contrast ratio of original colors). Thus, additional conversion of these colors is not performed, and the provisional colors are considered to be the final colors.

In example 2-2, the original background and foreground colors are \#EAE6E7 and \#6E6C6C, respectively, and the contrast ratio of the colors is 4.22 , whereas the provisional colors are \#FFD1D1 and \#7F878F, respectively, and the contrast ratio is 2.65. The contrast ratio of the provisional color is smaller than both 4.5 and the contrast ratio of the original colors (4.22). Therefore, the colors must be additionally converted. For the provisional colors, the relative luminance of background and foreground colors are calculated as 0.715 and 0.238 , respectively, by formulas (5)-(7). In addition, the saturation values of the background and foreground colors are 0.180 and 0.112 . Because the background color is lighter than the foreground color, and the saturation levels of both the background and foreground colors are low as determined in Section V-B1, the background and foreground colors are shifted to a lighter and darker color, respectively, in the low saturation level. Figure 2 shows the conversion process. The colors in this figure in the low saturation level are sorted by relative luminance. The additional conversion process is as follows:

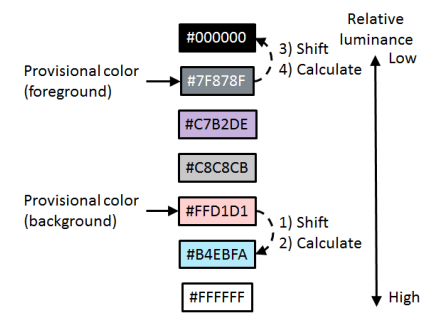

Fig. 2. Additional conversion process of example 2-2

1) Background color is shifted to the lighter color

2) Contrast ratio is calculated $\rightarrow 2.81$ (smaller than the threshold 4.22)

$\rightarrow$ Additional conversion is required $\rightarrow$ Go to 3 )

3) Foreground color is shifted to the darker color

4) Contrast ratio is calculated $\rightarrow 16.20$ (bigger than the threshold 4.22)

$\rightarrow$ Conversion is finished

When the background and foreground colors are shifted to \#B4EBFA and \#000000, respectively, the calculated contrast ratio is 16.20 , which is greater than 4.22 . Thus, the additional conversion is finished, and the shifted colors are considered to be the final colors.

In example 2-3, the provisional background and foreground colors become the same. Because the lighter and darker colors cannot be determined in this case, the relative luminance of the original background and foreground colors are used. The calculated relative luminance of the original background and foreground colors are 0.047 and 0.746 , respectively. Thus, because the background of the original color is darker than the foreground, the background and foreground of the provisional colors are shifted to the darker and lighter colors, respectively. In this example, the saturation level of both original colors 
were determined as 'high' in Section V-B1; accordingly, the provisional colors are converted to colors in the high saturation level. Figure 3 shows the conversion process. Colors in this figure in the high saturation level are sorted by relative luminance. The additional conversion process is as follows:

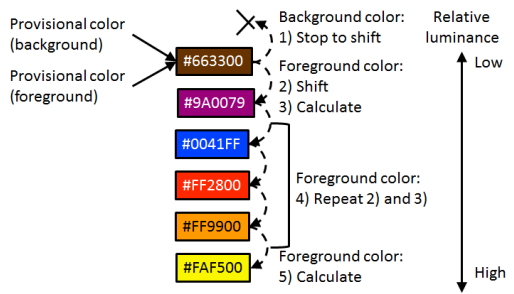

Fig. 3. Additional conversion process of example 2-3

1) Background of provisional color is the darkest color of this saturation level

$\rightarrow$ Background color cannot be shifted

2) Foreground of provisional color is shifted to the lighter color

3) Contrast ratio is calculated

$\rightarrow$ Smaller than threshold 8.20

$\rightarrow$ Additional conversion is required $\rightarrow$ Go to 4 )

4) 2) and 3) are repeated until the color \#FAF500 (until the contrast ratio become greater than 8.20)

5) Contrast ratio is calculated

$\rightarrow 8.89$ (bigger than threshold 8.20)

$\rightarrow$ Conversion is finished

When the foreground color is shifted to \#FAF500, the calculated contrast ratio is 8.89 and becomes greater than 8.20. Thus, the additional conversion is finished, and the shifted colors are considered to be the final colors.

\section{CONVERSION OF DOCUMENT COLORS}

\section{A. Conversion algorithm}

When background and foreground colors are converted, they do not always correspond one-to-one in the document. When document structures are represented by tree structures in which nodes represent blocks (parts of contents in documents) and edges represent inclusion of blocks, parent blocks often have some child blocks. This means that the size of parent blocks are bigger than child blocks. In this case, if the colors of a parent block are changed along with a child block, it may not be possible to convert colors of other child blocks appropriately. Thus, colors of parent blocks should be fixed when converting colors of child blocks. Document colors are converted by the algorithm shown in Fig. 4.

First, a tree is constructed from the target document. In the tree, nodes represent blocks, and edges represent the arrangement of child blocks on parent blocks. A block is extracted from the tree by depth-first search. If background or foreground colors are not assigned to the block, the colors are inherited from the parent block. If background (foreground) color is inherited, background (foreground) color is fixed, and foreground (background) color is converted by the conversion strategies described in Section V. Because colors of parent blocks are converted before conversion of the colors of child blocks, the fixed colors are the final colors of the parent blocks. If neither background nor foreground colors are inherited, both of them are converted.

\section{B. Example of converting document colors}

Figure 5 shows an example of the document structure at left and center. This example document is written by Hyper Text Markup Language (HTML). The source is shown at left, and the tree of the document is shown at center. In this document, blocks "h1," "h2," and "p" are arranged on the block "body." Background and foreground colors of the blocks are shown at right in Fig. 5. Because the background colors of blocks "h1" and "h2" are not given, the background color of the block "body" is applied to the background color of the block "h1" and "h2." In this case, if both the background and foreground colors of block "h1" are converted, the background color of the block "body" is also converted. This may result in an inappropriate combination between the background and foreground colors of block "h2." Thus, when the colors of block "h1" are converted, the background color is fixed.
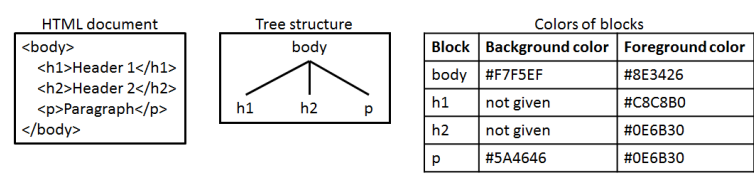

Fig. 5. Example of document structure

Applying the algorithm in Fig. 4, the colors of document are converted. First, the colors of block "body" is converted. Because block "body" does not have a parent block, both background and foreground colors are converted by the conversion strategies described in Section V. The final colors of the background and foreground colors of block "body" are \#FFD1D1 and \#663300, respectively.

Second, the colors of block "h1" are converted. Because the background color is not given, the background color of block "body" is applied. Thus, the additional conversion of the background color is not performed, according to the algorithm in Fig. 4, and only the foreground color of block "h1" is converted by the conversion strategies described in Section V. The final colors of the background and foreground of block "h1" are \#FFD1D1 (the final color of the background of the block "body") and \#C7B2DE, respectively.

Third, the colors of block "h2" are converted. As for block "h1," the background color is not given, but the foreground color is given. Thus, the conversion process is similar to that of block "h1." The final colors of the background and foreground of block "h2" are \#FFD1D1 (the final color of the background of the block "body") and \#663300, respectively.

Finally, the colors of block "p1" is converted. Because both the background and foreground colors are given, both of them are converted by the conversion strategies described in Section 


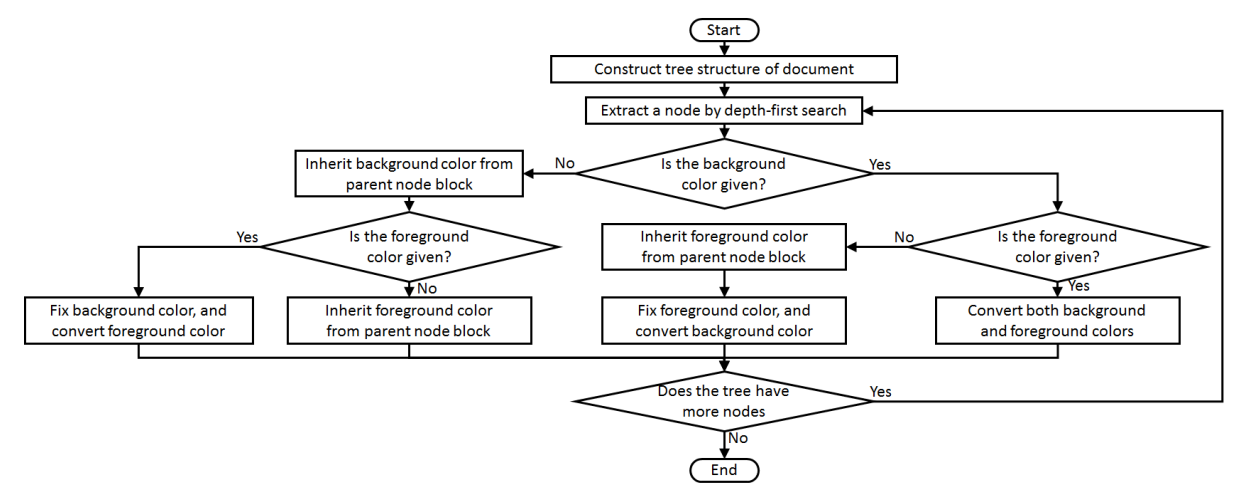

Fig. 4. Algorithm for converting document colors

$\mathrm{V}$. The final colors of the background and foreground of block "p" are \#7F878F and \#663300, respectively. Figure 6 shows the document before and after conversion.

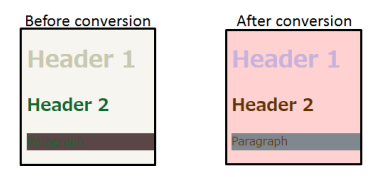

Fig. 6. Document colors before and after conversion

\section{EVALUATION}

To evaluate the validity of our method, we converted the colors of five web pages, and then our study subjects read them and answered questions.

\section{A. Procedures}

Five existing web pages were selected according to the following criteria:

1) To confirm distinguishability, more than one pair of background and foreground colors were used, with the exception of body colors.

2) To confirm attractiveness, more than one color with high saturation was used, with the exception of 1)

Although the selected web pages used image files to represent texts, our method is not designed to convert the colors of images, as mentioned in Section I. Therefore, we created web pages similar to the five selected using HTML and CSS files, and used the newly created web pages for this evaluation. As much as possible, image files were not used in the newly created web pages, and their appearances were almost the same as the original web pages. The existing web pages used for this evaluation were as follows (all are Japanese):

- Page A: http://www.ipa.go.jp/jinzai/asia/index.html (Referred on 19th December, 2016)

- Page B: https://www.nig.ac.jp/color/index.html (Referred on 19th December, 2016)

- Page C: http://www.dialoginthedark.com/today_ticket/ (Referred on 19th December, 2016)
- Page D: http://www-it.sci.waseda.ac.jp/TOP/ (Referred on 19th December, 2016)

- Page E: http://www.wul.waseda.ac.jp/imas/index.html (Referred on 19th December, 2016)

The colors of the created web pages were converted using our method, and screenshots of the color converted web pages were taken. Simulations of the appearances of these pages to color-impaired people with D-type impairments were generated using ImageJ [26] and Vischeck [27]. ImageJ is an image-processing application, and Vischeck is an application that can simulate files as they appear to color-impaired people. Vischeck can be used as plug-in for ImageJ. Then, subjects viewed the simulated screenshots and answered questions. Because the subjects saw the simulated screenshots, the contents of web pages did not change dynamically, and the web pages did not switch to other pages via hyperlinks.

A total of 12 subjects, all university students in their 20 s, took part in this evaluation. The evaluation conditions, including the order of presentation of the five web pages, the size of presentation, the computers used, and the display brightness, were the same for all subjects, and time limits were not set. Subjects answered four questions about each page by circling the appropriate text in response to the questions on the answer sheets. Answer sheets were printed for the five target web pages. Because the target web pages were printed in black and white on the answer sheets, subjects could not perceive the original attractive and distinguishable parts of the text from the answer sheets. The four questions were as follows:

Q1: Please circle the top three most attractive parts of the text, in terms of colors, and write their ranks in the blank spaces.

Q2: Please circle the top three least attractive parts of the text, in terms of colors, and write their ranks in the blank spaces.

Q3: Please circle the top three most distinguishable parts of the text, in terms of colors, and write their ranks in the blank spaces.

Q4: Please circle the top three least distinguishable parts of the test, in terms of colors, and write their ranks in the blank spaces. 


\section{B. Results}

Contrast ratios of each pair of background and foreground colors and saturation values of each color were calculated for the original five web pages, and the top three most attractive (high saturation values), least attractive (low saturation values), most distinguishable (high contrast ratio of relative luminance), and least distinguishable (low contrast ration of relative luminance) parts of the text were determined. These parts of the text were called "determined texts". The subjects' answers to questions Q1-Q4 were compared with the most attractive, least attractive, most distinguishable, and least distinguishable parts of the text, and the equivalence between the determined texts and the answers were confirmed.

Meanwhile, some of the original web pages contained images that could not be represented by HTML and CSS files, and these images remained in the newly created web pages. When the subjects answered the four questions, we explained that the images were not the target of this evaluation. However, there were answers in which the images were circled. Examples of these images were logo texts, which were difficult for the subjects to perceive them as images. Thus, we compared the determined texts with the answers in two cases: one in which answers including images were not counted ('no count' case), and another in which answers including images were evaluated as distinct from the determined texts ('distinct' case). The results are shown in Tables VI and VII.

TABLE VI

EQUIVALENCE OF ANSWERS ('NO COUNT' CASE)

\begin{tabular}{|l|l|l|l|l|}
\hline & \multicolumn{4}{|c|}{ Rates of identicalness (\%) } \\
\cline { 2 - 5 } & $\begin{array}{l}\text { Q1: Most } \\
\text { attractive }\end{array}$ & $\begin{array}{l}\text { Q2: Least. } \\
\text { attractive }\end{array}$ & $\begin{array}{l}\text { Q3: Most } \\
\text { distinguishable }\end{array}$ & $\begin{array}{l}\text { Q4: Least } \\
\text { distinguishable }\end{array}$ \\
\hline Page A & 65.0 & 44.1 & 56.3 & 25.0 \\
\hline Page B & 88.5 & 58.3 & 34.4 & 31.4 \\
\hline Page C & 76.7 & 51.6 & 46.7 & 71.0 \\
\hline Page D & 70.0 & 47.1 & 65.0 & 12.1 \\
\hline Page E & 48.0 & 75.0 & 66.7 & 37.1 \\
\hline Total & 70.3 & 55.6 & 52.1 & 34.9 \\
\hline
\end{tabular}

TABLE VII

EQUIVALENCE OF ANSWERS ('DISTINCT' CASE)

\begin{tabular}{|l|l|l|l|l|}
\hline & \multicolumn{4}{|c|}{ Rates of identicalness (\%) } \\
\cline { 2 - 5 } & $\begin{array}{l}\text { Q1: Most } \\
\text { attractive }\end{array}$ & $\begin{array}{l}\text { Q2: Least. } \\
\text { attractive }\end{array}$ & $\begin{array}{l}\text { Q3: Most } \\
\text { distinguishable }\end{array}$ & $\begin{array}{l}\text { Q4: Least } \\
\text { distinguishable }\end{array}$ \\
\hline Page A & 36.1 & 41.7 & 25.0 & 23.5 \\
\hline Page B & 65.7 & 58.3 & 27.8 & 31.4 \\
\hline Page C & 63.9 & 44.4 & 38.9 & 61.1 \\
\hline Page D & 19.4 & 44.4 & 36.1 & 11.1 \\
\hline Page E & 33.3 & 75.0 & 44.4 & 36.1 \\
\hline Total & 43.6 & 52.8 & 34.4 & 32.8 \\
\hline
\end{tabular}

The numbers in the tables are the rates at which the subjects' answers that were identical to the determined texts. Here, we only considered whether the answers were included in the top three determined texts; we did not evaluate the equivalence of individual rankings. Additionally, there were opinions that it was difficult to select answers because of few colors and factors except colors, such as text size, arrangement, text densities, and decorations.

\section{Discussion}

According to Tables VI and VII, the answer rates for Q1 and Q3 in the 'no count' case were clearly higher than those for Q2 and Q4 in the 'distinct' case. This means that the subjects selected images as the answers for Q1 and Q3 more often than for Q2 and Q4. Because images are not the targets of our method, this represents a problem of the evaluation procedure. To evaluate more appropriately, it is necessary to select web pages without images.

Although the rates of equivalence of answers for Q1 and Q3 in Table VI are higher than those in Table VII, the rates for Q2 and Q4 are similar in both tables. This indicates that it might be difficult for the subjects identify less distinguishable and less attractive parts of the text.

Meanwhile, the rates of equivalence for answers for Q1 and Q2 were higher than those for Q3 and Q4, respectively, in both the 'no count' and 'distinct' cases. Q1 and Q2 are questions related to attractiveness, whereas Q3 and Q4 are related to distinguishability. There were many common answers between Q1 (Q2) and Q3 (Q4). This indicates that attractiveness is related to distinguishability, and that the sizes and arrangements of text, in addition to color, affected attractiveness and distinguishability.

The answers that were not identical to the determined texts for Q1 and Q3 included headlines and itemized sentences. For Q2 and Q4, parts of the text that were small, or had narrow line spacing, were selected. These findings indicate that conditions other than colors were reflected in the answers.

In addition, the contrast ratios for some pairs of final background and foreground colors became significantly higher than the contrast ratios for their original colors. Due to this, the distinguishability of the parts of the text with the final colors might have been higher than those of the parts with the original colors. If the designers' intentions are considered, the conversion strategies should be improved in order to not make the contrast ratios too high.

However, the total rate of equivalence for answers for Q1 was over $70 \%$ for 'no count' case, indicating that the parts of the text with high attractiveness could be perceived. This result confirms the effectiveness of our method.

\section{Threats to validity}

In this evaluation, the converted colors of the target web pages were simulated for D-type color impairment. As noted in Section III-A, there are other types of color impairment, including P-type, T-type, and A-type. Even though certain colors are simulated, the results of simulations are often quite different among these types. Thus, if the converted colors of the target web pages were simulated for other types of vision, the results might be different.

Meanwhile, the subjects were all C-type people, and the converted colors of web pages in the evaluation were simulated for vision of color-impaired people. However, the conditions 
of color-impaired people are individually different, even within a given type of color impairment. This means that evaluation by color-impaired people might yield different results.

As described in Section I, the target of our method is text in documents, rather than images. These documents include images, which are not converted. In addition, our method assumes that the text in a target document is divided into blocks like HTML tags, and each block has one background color and one foreground color. If a block has multiple background or foreground colors, our method cannot be applied.

In addition, our method assumes that designers use highsaturation colors for parts of the text that they want to make attractive, and pairs of background and foreground colors with high contrast ratios for parts of the text that they want to make distinguishable. However, if designers do not follow these color-use strategies, our method will not work appropriately.

\section{CONCLUSION}

This paper describes our method for converting document colors so that color-impaired people can perceive the designers' intentions, here defined as attractiveness and distinguishability. High attractiveness is represented as colors with high saturations, whereas high distinguishability is represented as pairs of background and foreground colors with high contrast ratios. The conversion is performed in two steps. The first conversion is performed using the basic conversion table, whereas the second conversion is performed by calculating contrast ratios of pairs of background and foreground colors. In the second conversion, if the calculated contrast ratios do not satisfy certain thresholds, additional conversions are performed. In addition, we propose an algorithm for applying these conversion strategies to documents.

To evaluate the method, five web pages were selected and the colors were simulated to the appearance of D-type color impairment after color conversion. Twelve subjects viewed the simulated web pages and answered four questions. The results showed that although conditions other than colors affected the answers, attractive parts of the text could be perceived. These findings indicated that our method was effective.

Future work will focus on the following:

- Improvement of evaluation procedures

- Considerations of various types of color impairments

- Evaluation by color-impaired people

- Increasing the number of candidate colors for conversion

\section{REFERENCES}

[1] Facts About Color Blindness. [Online]. Available: https://nei.nih.gov/ health/color_blindness/facts_about (Referred in May 6, 2017).

[2] Color Universal Design Colorset Guidebook. [Online]. Available: http://www2.cudo.jp/wp/wp-content/uploads/2016/10/CUD_Colorset_ Guidebook.pdf (in Japanese) (Referred in May 6, 2017).

[3] L. Zhou, V. Bensal, and D. Zhang, "Color adaptation for improving mobile web accessibility", Procs. of 2014 IEEE/ACIS 13th International Conference on Computer and Information Science (ICIS), Taiyuan, China, 2014. http://dx.doi.org/10.1109/ICIS.2014.6912149

[4] D. R. Flatla, K. Reinecke, C. Gutwin, and K. Z. Gajos, "SPRWeb: preserving subjective responses to website colour schemes through automatic recolouring", Procs. of the SIGCHI Conference on Human Factors in Computing Systems, Paris, France, pp. 2069-2078, 2013. http://dx.doi.org/10.1145/2470654.2481283
[5] L. C. Ou, M. R. Luo, A. Woodcock, and A. Wright, "A study of colour emotion and colour preference. Part I: Colour emotions for single colours", Color Research \& Application, Volume 29, pp. 232240, 2004. http://dx.doi.org/10.1002/col.20010

[6] H. Takimoto, H. Yamauchi, M. Jindai, and A. Kanagawa, "Modification of indistinguishable colors for people with color vision deficiency", Journal of Signal Processing Volume 16, Number 6, pp. 587-592, 2012. http://dx.doi.org/10.2299/jsp.16.587

[7] A. Kruger, "Median-cut color quantization”, Dr. Dobb’s Jounal, 1994.

[8] A. A. Gooch, S. C. Olsen, J. Tumblin, and B. Gooch, "Color2Gray: salience-preserving color removal", Proc. SIGGRAPH, Los Angeles, California, pp. 634-639, 2005. http://dx.doi.org/10.1145/1073204.1073241

[9] N. H. N. A. Wahab, F. S. Ismail, and M. A. A. Nawawi, "Color Transformation Method for Protanopia Vision Deficiency using Artificial Neural Network", Journal of Telecommunication, Electronic and Computer Engineering, Volume 8, Number 11, pp. 29-33, 2016.

[10] H. Orii, H. Kawano, N. Suetake, and H. Maeda, "Color conversion for color blindness employing multilayer neural network with perceptual model", Proc. of the 7th Pacific-Rim Symposium on Image and Video Technology (PSIVT 2015), Volume 9431, pp. 3-14, Auckland, New Zealand, 2015. http://dx.doi.org/10.1007/978-3-319-29451-3_1

[11] CIE, "Commission internationale de l'Eclairage proceedings", Cambridge University Press, 1931.

[12] T. Smith and J. Guild, "The C.I.E. colorimetric standards and their use", Transactions of the Optical Society, Volume 33, Number 3, pp. 73-134, 1932. http://dx.doi.org/10.1088/1475-4878/33/3/301

[13] V. C. Smith and J. Pokorny, "Spectral sensitivity of the foveal cone photopigments between 400 and $500 \mathrm{~nm}$ ", Vision Research, Volume 15, Issue 2, pp. 161-171, 1975. http://dx.doi.org/10.1016/00426989(75)90203-5

[14] F. Vienot, H. Brettel, and J. D. Mollon, "Digital video colourmaps for checking the legibility of displays by dichromats", Color Research and Application Volume 24, Issue 4, pp. 243-252, 1999. http://dx.doi.org/10.1002/(SICI)1520-6378(199908)24:4<243::AIDCOL5>3.0.CO;2-3

[15] N. Suetake and G. Tanaka, "Color transformation techniques for gamuts of lower dimension - color transformation for monochrome display/print and dichromacy -". IEICE ESS Fundamentals Review Volume 6, Number 2, pp. 102-113, 2012 http://dx.doi.org/10.1587/essfr.6.102

[16] R. Harwahyu, A. S. Manaf, B. S. Ananto, B. A. Wicaksana, and R. F. Sari, "Implementation of color-blind aid system", Journal of Computer Science, Volume 9, Number 6, pp. 794-810, 2013. http://dx.doi.org/10.3844/jcssp.2013.794.810

[17] S. Tajima and K. Komine, "Saliency-based color accessibility", IEEE Transactions on Image Processing, Volume 24, Number 3, pp. 1115 1126, 2015., http://dx.doi.org/10.1109/TIP.2015.2393056

[18] M. Geissbuehler and T. Lasser, "How to display data by color schemes compatible with red-green color perception deficiencies", Opt. Express 21, pp. 9862-9874, 2013. http://dx.doi.org/10.1364/OE.21.009862

[19] M. Tennekes and E. Jonge, "Tree colors: color schemes for treestructured data", IEEE Transactions on Visualization and Computer Graphics, Volume 20, Number 12, pp. 2072-2081, 2014. http://dx.doi.org/10.1109/TVCG.2014.2346277

[20] K. Tsuchida, C. Kato, and T. Nagshima, "Color barrier free displays in disaster situations", Open Journal of Social Sciences Volume 4 Number 2, pp. 25-29, 2016. http://dx.doi.org/10.4236/jss.2016.42005

[21] A. Frane, "A call for considering color vision deficiency when creating graphics for psychology reports", The Journal of General Psychology, Volume 142, Number 3, pp. 194-211, 2015. http://dx.doi.org/10.1080/00221309.2015.1063475

[22] A. R. Smith, "Color gamut transformation pairs", Computers Graphics vol.12, pp.12-19, 1978. http://dx.doi.org/10.1145/965139.807361.

[23] Japan Color Research Institute, "Practical color co-ordinate system", Japan Color Research Institute, 1964.

[24] Japan Color Research Institute, "PCCS harmonic color chart 201-L", Japan Color Research Institute, 1999.

[25] Web Content Accessibility Guidelines 2.0. [Online]. Available: https://www.w3.org/TR/WCAG20/ (Referred in May 6, 2017).

[26] ImageJ. [Online]. Available, https://imagej.nih.gov/ij/ (Referred in May $6,2017)$

[27] VischeckJ. [Online]. Available, http://www.vischeck.com/ (Referred in May 6, 2017) 